\title{
A porcine reproductive and respiratory syndrome virus (PRRSV) vaccine candidate based on the fusion protein of PRRSV glycoprotein 5 and the Toll-like Receptor-5 agonist Salmonella Typhimurium FljB
}

Dan Xiong ${ }^{1,2+}$, Li Song ${ }^{1,2+}$, Xianyue Zhai ${ }^{1,2}$, Shizhong Geng ${ }^{1,2}$, Zhiming Pan ${ }^{1,2^{*}}$ and Xinan Jiao ${ }^{1,2^{*}}$

\begin{abstract}
Background: Porcine reproductive and respiratory syndrome (PRRS) is characterized by severe reproductive failure and severe pneumonia in neonatal pigs and is caused by PRRS virus (PRRSV). Glycoprotein 5 (GP5) from PRRSV is a key inducer of neutralizing antibodies. Flagellin, a toll-like receptor 5 (TLR-5) agonist, is an effective inducer of innate immune responses. This study presents a novel PRRSV vaccine candidate based on the adjuvant effect of Salmonella Typhimurium FljB fused with PRRSV GP5.

Results: A truncated rGP5 gene lacking the signal peptide and transmembrane sequences was amplified and inserted into prokaryotic expression vectors, pColdl or pGEX-6p-1. Salmonella Typhimurium flagellin fljB was amplified and inserted into the plasmid pCold-rGP5, generating recombinant plasmid pCold-rGP5-fljB. Histidine (His)-tagged rGP5 and fusion protein rGP5-FljB were induced with isopropyl- $\beta$ - $d$-thiogalactoside, verified by SDS-PAGE and western blotting, and purified via Ni-NTA affinity columns. The TLR-5-specific bioactivity of fusion protein rGP5-FljB was determined by detecting the expression levels of the cytokine IL-8 in HEK293-mTLR5 cells by sandwich ELISA. The purified endotoxin-free proteins were administered intraperitoneally in a $\mathrm{C} 3 \mathrm{H} / \mathrm{HeJ}$ mouse model. The results show that immunization with the fusion protein rGP5-FljB induced a significantly enhanced GP5-specific and PRRSV-specific IgG response that persisted for almost 5 weeks. Co-administration of the rGP5 with R848 or Alum also yielded a higher lgG response than administration of rGP5 alone. The lgG1/lgG2a ratio in the rGP5-FljB immunization group was significantly higher (9-fold) than that in the rGP5 alone group and was equivalent to the response in the rGP5 + Alum immunization group, suggesting a strong Th2 immune response was induced by the fusion protein.
\end{abstract}

Conclusions: Purified fusion protein rGP5-FljB is capable of activating the innate immune response, as demonstrated by the results of our TLR-5-specific bioactivity assay, and FljB has adjuvant activity, as shown by the results from our administration of rGP5-FljB in a mouse model. Our findings confirm that FljB could serve as an excellent adjuvant for the production of GP5-specific and PRRSV-specific lgG antibodies as part of an induction of a robust humoral immune response.

Keywords: PRRSV, Glycoprotein 5, Flagellin, Fusion protein, Vaccine

\footnotetext{
*Correspondence: zmpan@yzu.edu.cn; jiao@yzu.edu.cn

${ }^{\dagger}$ Equal contributors

'Jiangsu Key Laboratory of Zoonosis, Yangzhou University, 48 East Wenhui

Road, Yangzhou, Jiangsu 225009, China

${ }^{2}$ Jiangsu Co-innovation Center for Prevention and Control of Important

Animal Infectious Diseases and Zoonoses, Yangzhou, Jiangsu 225009, China
} 


\section{Background}

Porcine reproductive and respiratory syndrome (PRRS) has been recognized as one of the most serious infectious diseases of swine since its first appearance in North America in 1987 [1]. The disease is characterized by severe reproductive failure and severe pneumonia in neonatal pigs and is caused by PRRS virus (PRRSV), a member of the Arteriviridae family, order Nidoviridales [2].

Pigs mount a rapid antibody response to infection by PRRSV, but the antibodies are mainly directed to the $\mathrm{N}$ - and M-proteins and are non-neutralizing [3]. The primary neutralization epitope of some North American PRRSVs is located in the middle of the glycoprotein 5 (GP5) ectodomain [4]. A truncated GP5 without the signal peptide sequence or the predicted transmembrane regions (residues 60-130) is able to elicit protective antibodies capable of detecting PRRSV-infected cells and of distinguishing this virus from others [5]. Currently, killed-virus and modified-live PRRSV vaccines are used to control PRRS. However, both of these types of vaccines have inherent drawbacks and the development of novel PRRSV vaccines is urgently needed $[6,7]$.

Recent advances in innate immunity research have indicated that pathogen-associated molecular patterns (PAMPs) are promising molecular adjuvants for subunit vaccines $[8,9]$. Flagellin, the structural component of the flagellar filament in various locomotive bacteria, is a ligand for toll-like receptor 5 (TLR-5) in host cells $[10$, 11]. An increasing number of studies have demonstrated the effectiveness of flagellin as an adjuvant $[12,13]$, and flagellin is an effective inducer of innate immune effectors, such as cytokines and nitric oxide, thereby stimulating the activation of adaptive immune responses [14].

In the present study, we cloned a truncated rGP5 gene and constructed the fusion protein rGP5-FljB using a prokaryotic system. We then determined the TLR-5specific bioactivity of fusion protein rGP5-FljB by detecting the expression levels of the cytokine interleukin 8 (IL-8) in HEK293-mTLR5 cells by sandwich enzymelinked immunosorbent assay (ELISA). Last, we assessed the immunogenicity of rGP5 and the adjuvant properties of $\mathrm{FljB}$ in a mouse immunization assay. ELISA-based detection of GP5-specific and PRRSV-specific antibodies suggested that FljB could enhance the immunogenicity of GP5 and induce a robust humoral immune response, thus providing more effective antibodies against PRRSV.

\section{Results}

\section{Construction of expression plasmids bearing rGP5 and rGP5-fljB}

A truncated rGP5 gene lacking the signal peptide sequence and transmembrane regions was amplified using a linker-based overlap-PCR strategy. The gene of interest was inserted into the expression plasmids pColdI and pGEX-6p-1 to add a His or GST tag, respectively. Recombinant plasmid pCold-rGP5-fljB was constructed by using enzymes to digest the plasmid pCold-rGP5 and inserting the PCR product $f l j B$ (Fig. 1). The DNA sequencing results indicated that the sequences of the inserts were identical to the template sequences.

\section{Expression and purification of rGP5 and rGP5-FljB proteins}

The expression of His-rGP5, GST-rGP5, and rGP5-FljB was induced by addition of IPTG to the culture medium. SDS-PAGE results indicated that the molecular weights of the tagged proteins were about $15 \mathrm{kDa}$ for His-rGP5, $40 \mathrm{kDa}$ for GST-rGP5, and $67 \mathrm{kDa}$ for rGP5-FljB, as expected (Fig. 2). His-rGP5 and rGP5-FljB were purified via a Ni-NTA affinity column and verified by SDS-PAGE analysis (Fig. 3).

\section{Immunoblotting}

To analyze the immunoreactivity of the rGP5 and fusion protein $\mathrm{rGP5}$-FljB, polyclonal antibodies against PRRSV or $\mathrm{FljB}$ were used as the primary antibodies in a western blotting assay. The results showed that the bacteriallyexpressed rGP5 and rGP5-FljB were able to react with the PRRSV-specific antibody (Fig. 4a). Additionally, western blotting with anti-FljB antibodies confirmed the presence of a highly purified $67 \mathrm{kDa}$ protein corresponding to rGP5-FljB (Fig. 4b).

\section{TLR-5 activation by purified fusion protein rGP5-FljB}

After removal of endotoxin from the purified proteins rGP5 and rGP5-FljB, very low endotoxin levels, less than $0.03 \mathrm{EU} / \mu \mathrm{g}$, were detected in the protein preparations, indicating that LPS contamination in the prepared proteins was negligible. To verify the activation of innate immune signaling by fusion protein $\mathrm{rGP} 5-\mathrm{FljB}$, we conducted a TLR-5-specific bioactivity assay. Stimulation with rGP5-FljB at several different protein concentrations elicited a robust production of IL-8 that was significantly higher than that elicited by stimulation with similar concentrations of rGP5 alone $(p<0.01$; Fig. 5). The observed up-regulation of chemokine IL- 8 following stimulation with $\mathrm{rGP5}-\mathrm{FljB}$ suggested that the prepared endotoxin-free $\mathrm{rGP} 5$-FljB was able to activate the innate immune response in vitro.

\section{Effects of rGP5-FljB treatment on GP5-specific and PRRSV-specific serum antibodies}

To examine the antibody response to the recombinant protein in an animal model, we administered the endotoxin-free fusion protein $\mathrm{rGP} 5-\mathrm{FljB}$ via an intraperitoneal injection. The results showed that immunization with the fusion protein induced a significantly enhanced GP5-specific and PRRSV-specific IgG response after 


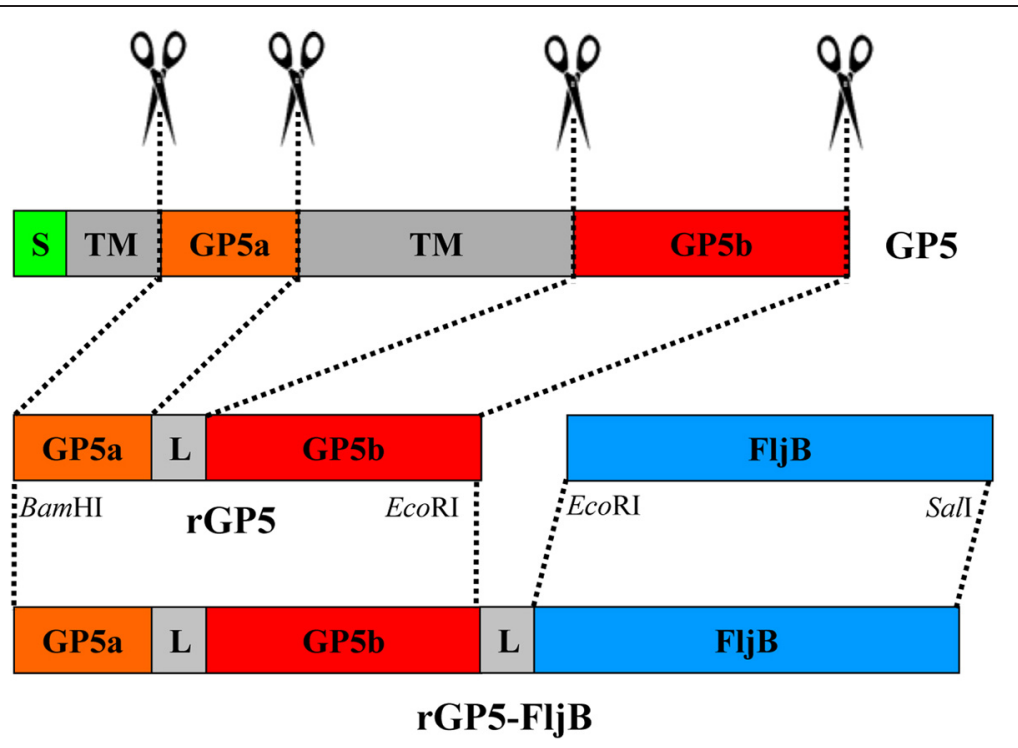

Fig. 1 Schematic representation for the construction of rGP5 and rGP5-fljB. rGP5 fragment with the deletion of its signal peptide (green) and transmembrane regions (light grey) was amplified by overlap-PCR, and inserted into the BamHI and EcoRI digested expression vector pColdl or pGEX-6p-1 to create pCold-rGP5 or pGEX-6p-1-rGP5 respectively. Flagellin fljB gene (light blue) was amplified from the genomic DNA of attenuated Salmonella Typhimurium SL7207 strain and cloned into the EcoRI and Sall sites of pCold-rGP5, resulting in a recombinant plasmid pCold-rGP5-fljB. "L" represents the linker sequence GGGGS

three immunizations compared with immunization using rGP5 alone (Figs. 6b and 7). The titer in the rGP5-FljB immunization group was elevated almost four-fold after the third immunization compared with the titer after the second immunization. Co-administration of the rGP5 with R848 or Alum also yielded a higher IgG response than immunization with rGP5 alone (Fig. 6b).

\section{Immune subtype induced by fusion protein rGP5-FljB}

We investigated the subtype of the immune response after three immunizations with fusion protein rGP5FljB by detecting serum IgG1 and IgG2a via indirect
ELISA. Co-administration of rGP5 with R848 or Alum induced a robust Th2 immune response. The IgG1/IgG2a ratio in the group of mice immunized with rGP5 alone was 4.0, whereas in the group of mice immunized with rGP5 together with R848 or Alum this ratio was 11.8 or 35.5 , respectively. The IgG1/IgG2a ratio in the rGP5-FljB immunization group was significantly higher (9-fold) than that in the rGP5 alone immunization group, and reached a level equivalent to the IgG1/IgG2a ratio in the rGP5 + Alum immunization group (Fig. 6c), suggesting a strong Th2 immune response.

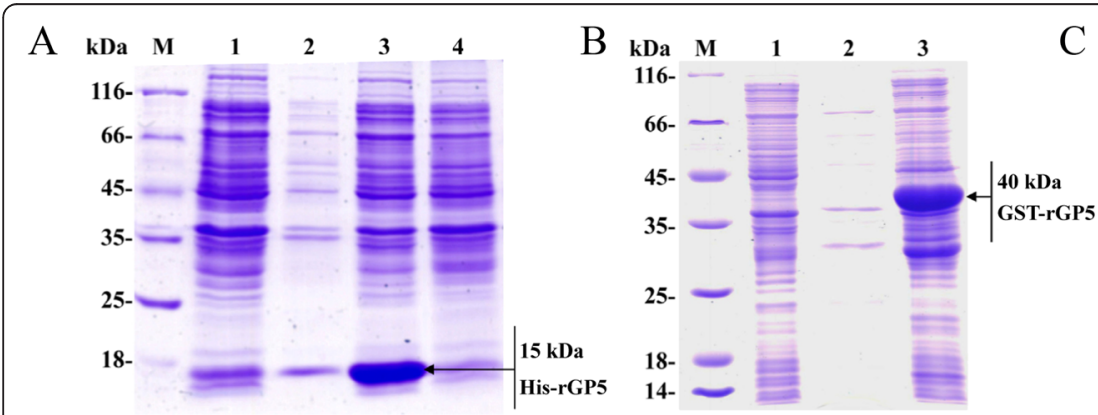

Fig. 2 SDS-PAGE analysis of recombinant bacteria of BL21 (DE3)(pCold-rGP5) (a), BL21(DE3)(pGEX-6p-1-rGP5) (b) and BL21 (DE3)(pCold-rGP5-fljB) (c). (a) Lanes: M, molecular weight markers; 1, product of BL21(DE3)(pCold) induced by IPTG; 2, Lysate supernatant of bacteria bearing His-rGP5 induced by IPTG; 3, Inclusion bodies of bacteria bearing His-rGP5 induced by IPTG; 4, product of bacteria bearing His-rGP5 not induced. (b) Lanes: M, molecular weight markers; 1, product of BL21(DE3)(pGEX-6p-1) induced by IPTG; 2, Lysate supernatant of bacteria bearing GST-rGP5 induced by IPTG; 3 , Inclusion bodies of bacteria bearing GST-rGP5 induced by IPTG. (c) Lanes: M, molecular weight markers; 1, product of BL21(DE3)(pCold) induced by IPTG; 2 , product of bacteria bearing rGP5-FljB not induced; 3, Lysate supernatant of bacteria bearing rGP5-FljB induced by IPTG; 4, Inclusion bodies of bacteria bearing rGP5-FljB induced by IPTG 

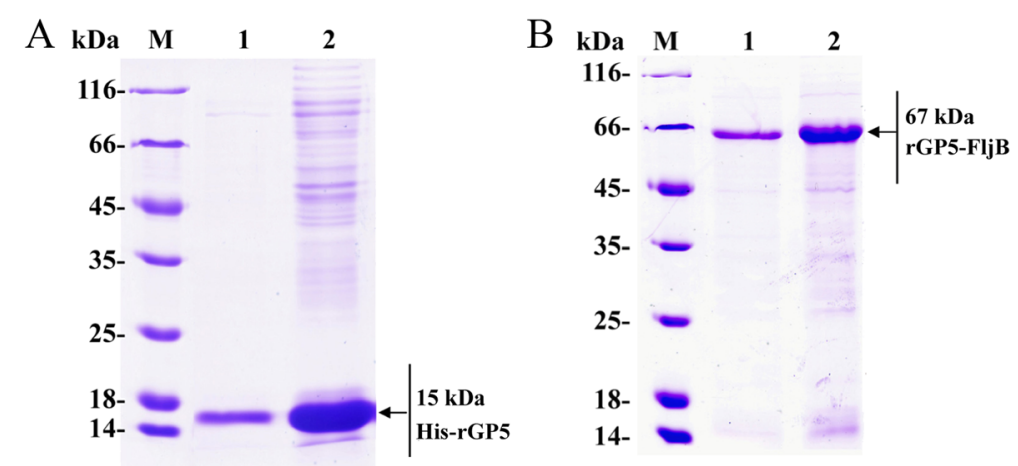

Fig. 3 SDS-PAGE analysis of purified His-rGP5 and rGP5-FljB proteins. The fused His-rGP5 (a) and rGP5-FljB (b) were purified via Ni-NTA affinity columns. Lanes: M, molecular weight markers; 1 and 2, purified His-rGP5 or rGP5-FljB proteins

\section{The longevity of GP5-specific serum IgG}

To monitor the longevity of the GP5-specific serum IgG, immunized animals were bled at 12-day intervals for 36 days after the third immunization and serum IgG levels were determined by indirect ELISA. The ELISA plates were coated with GST-tagged GP5 antigen. We analyzed the time course of the levels of GP5-specific antibodies induced by rGP5 alone, rGP5-FljB, or rGP5 + R848 (Fig. 8). The IgG titer in the rGP5-FljB group reached its peak $(11,520)$ on day 12 post-third immunization, and then gradually fell. The rGP5-FljB immunization induced an approximately 7-12 fold increase in the GP5-specific IgG titers compared with the rGP5 alone immunization over the different tested time points, and the IgG titer of the rGP5-FljB group was still significantly higher than that of the rGP5 alone group on day 36 post-third immunization. These results suggest that rGP5-FljB immunization produces both a higher and longerlasting anti-GP5 IgG titer than immunization with rGP5 alone.

\section{Discussion}

PRRSV infection has been recognized as a severe threat to the pig industry. A hallmark of the swine antibody response against PRRSV is the abundant nonneutralizing antibodies detected early in infection followed by a low neutralizing antibody titer that appears at least 3 weeks after infection $[15,16]$. The viral glycoprotein can induce a potent humoral immune response, and the production of anti-GP5 antibody is associated with the disappearance of viremia [4].

The Escherichia coli (E. coli)-based prokaryotic expression system is a powerful host cell system for expressing heterologous genes [17]. Many glycoproteins from cells or viruses produced in this system have shown biological activities [18, 19]. Therefore, we aimed to express a truncated rGP5 gene [5] without the signal peptide sequence and transmembrane regions in E. coli. The fused His-rGP5 was purified via a Ni-NTA affinity column by its His-tag (Fig. 3a). The immunoreactivity of rGP5 was determined by western blotting, and the results show that the heterologously-expressed protein reacts with an
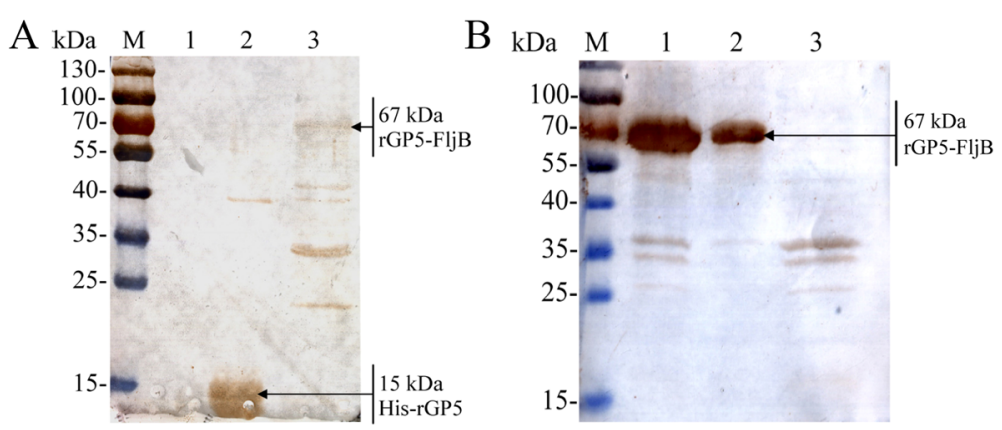

Fig. 4 Western blotting analysis of His-rGP5 and rGP5-FljB proteins. (a) Analysis of His-rGP5 and rGP5-FljB with an anti-PRRSV polyclonal antibody. Lanes: M, molecular weight markers; 1, $1 \times$ SDS-loading buffer; 2, purified His-rGP5 protein; 3, purified rGP5-FljB protein. (b) Analysis of rGP5-FljB with an anti-FljB polyclonal antibody. Lanes: M, molecular weight markers; 1, Lysate supernatant of BL21(DE3) (pCold-rGP5-FljB) induced by IPTG; 2, Inclusion bodies of BL21(DE3)(pCold-rGP5-FljB) induced by IPTG; 3, product of BL21(DE3)(pCold) induced by IPTG 


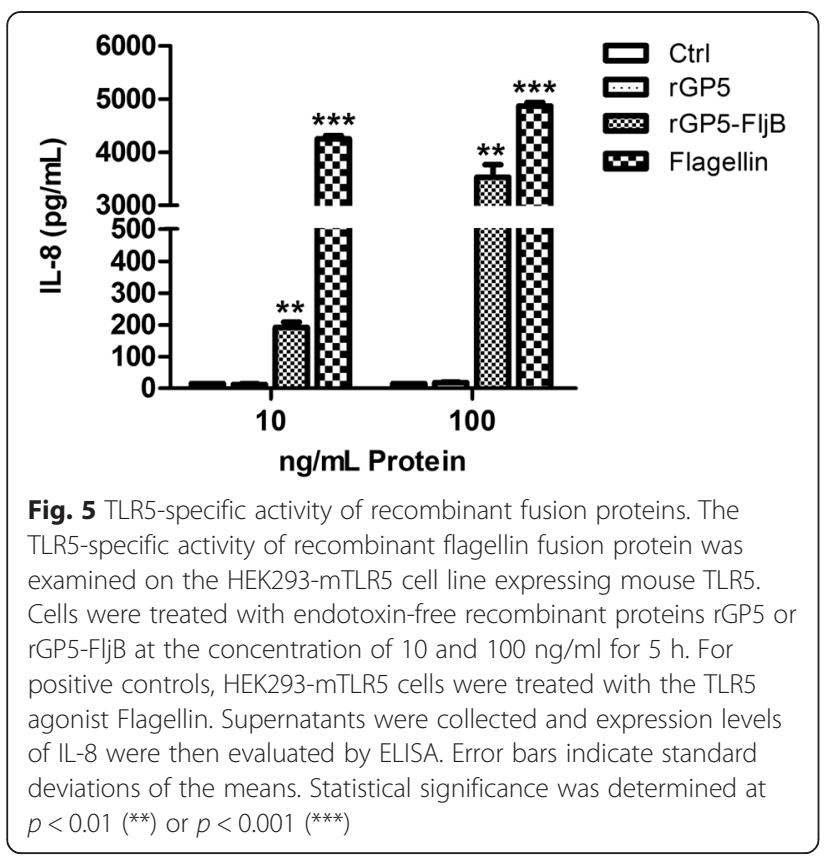

anti-PRRSV polyclonal antibody (Fig. 4a), indicating that the recombinant protein retained the biological activity of the wild-type protein.

TLRs recognize distinctive ligands, play key roles in innate immunity, and can contribute to the development of appropriate adaptive immune responses [20]. Recently, several TLR ligands have been widely studied as adjuvants for immunotherapy and vaccination $[8,21]$. Among the

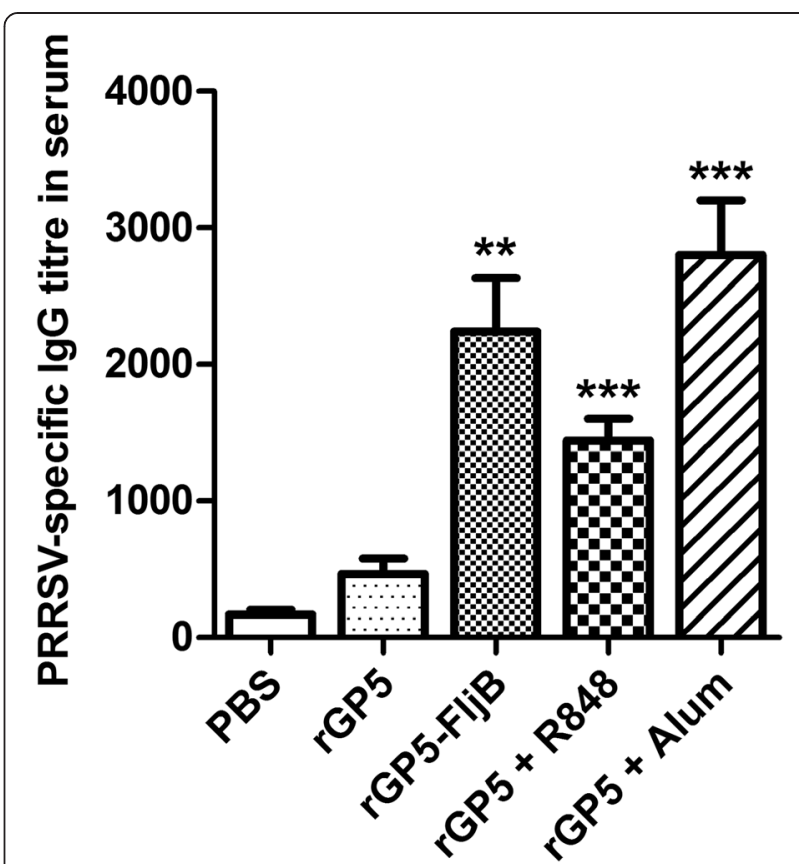

Fig. 7 PRRSV-specific IgG antibody titers in serum at day 12 after the third immunization. $\mathrm{C} 3 \mathrm{H} / \mathrm{HeJ}$ mice were randomly divided into five groups (6 mice per group) and immunized intraperitoneally either with rGP5, rGP5-FljB, rGP5 + R848, rGP5 + aluminium adjuvant, or $P B S$, respectively. These mice were immunized three times on days 0, 14, and 28 at a dose of $50 \mu \mathrm{g}$ rGP5, $50 \mu \mathrm{g}$ rGP5-FljB, $10 \mu \mathrm{g}$ R848 or isochoric aluminium adjuvant in $200 \mu \mathrm{L}$. Blood was collected from eye sockets on days 40 for analysis of anti-PRRSV IgG titers by ELISA. Data reflects the mean \pm SD by using the Student $t$ test at $p<0.01$ $\left({ }^{* *}\right)$ or $\left.p<0.001{ }^{* * *}\right)$

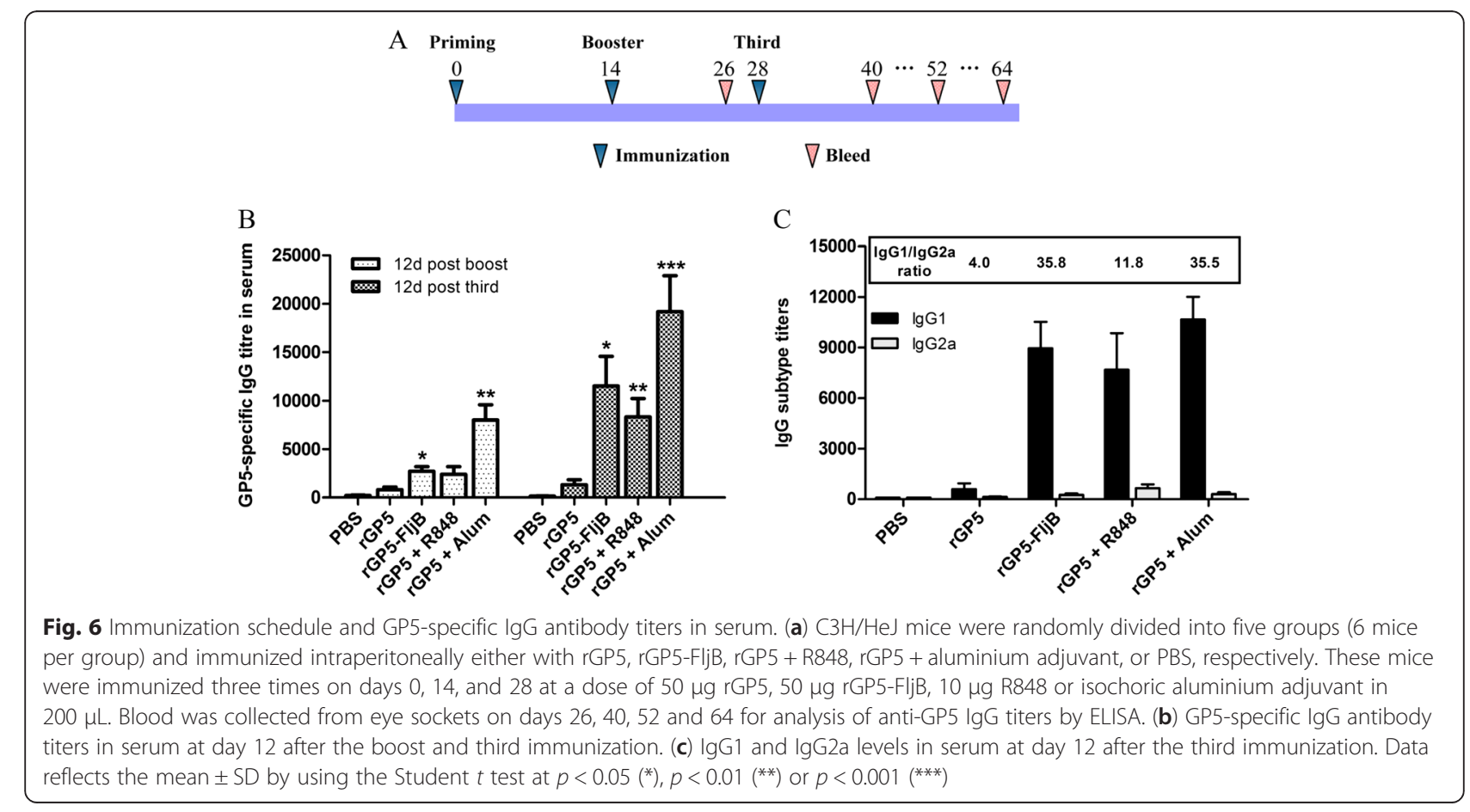




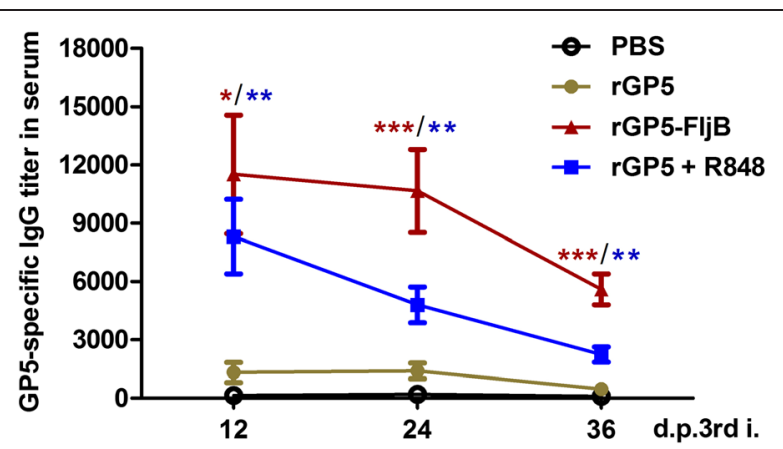

Fig. 8 The longevity of GP5-specific serum lgG titers. Mice were bled for 36 days at 12 days intervals after 3rd immunization, and serum IgG levels were determined by indirect ELISA. The ELISA plates were coated with GST-taged GP5 antigen. The time course of GP5-specific antibodies induced by rGP5 alone, rGP5-FljB or rGP5 + R848 was analyzed. Data reflects the mean \pm SD by using the Student $t$ test at $\left.p<0.05\left(^{*}\right), p<0.011^{* *}\right)$ or $p<0.0011^{(* *)}$

defined TLR agonists, flagellin is the only protein with defined genetic codes [22]. To investigate the adjuvant activity of fusion protein rGP5-FljB, phase II flagellin $f l j B$ was amplified from the S. Typhimurium SL7207 strain and the recombinant plasmid pCold-rGP5-fljB was constructed. The fusion protein preparation had a high degree of purity, as only one protein band was observed (Fig. 3b). Flagellins from Gram-negative bacteria undergo a direct interaction with the leucine-rich regions in TLR-5 and activate a range of inflammatory cells via a TLR-5dependent signaling pathway $[11,12]$. We conducted a TLR-5-specific bioactivity assay and the results showed that stimulation with fusion protein $\mathrm{rGP} 5-\mathrm{FljB}$ induced a significantly higher expression of IL-8 than stimulation with rGP5 alone, at several different protein concentrations (Fig. 5), indicating that the innate immune response was activated by flagellin FljB.

Recently, an ever increasing number of studies have described the adjuvant property of flagellin in the context of a broad range of recombinant vaccines [23, 24]. We tested the ability of rGP5-FljB to act as an adjuvant by administering the fusion protein $\mathrm{rGP} 5-\mathrm{FljB}$ to mice, and found that immunization with the fusion protein induced a significantly-enhanced GP5-specific and PRRSVspecific IgG response compared with immunization with rGP5 alone (Figs. 6b and 7), indicating that the polyclonal antibodies against rGP5 were able to react with PRRSV and $\mathrm{FljB}$ is an efficacious adjuvant for the induction of antigen-specific IgG production.

Imiquimod, a synthetic TLR-7 agonist, can expedite the immune response against influenza virus infection when combined with influenza vaccines [25]. In this study, the GP5-specific IgG titer following rGP5 + R848 co-administration was significantly upregulated after the third immunization (Fig. 6b) and persisted for almost 5 weeks (Fig. 8). The robust IgG1/IgG2a ratio post-third immunization in the rGP5-FljB group, similar to that of the rGP5 + R848/Alum co-administration groups, indicated that a strong GP5-specific Th2 response was induced by the fusion protein $\mathrm{rGP5}-\mathrm{FljB}$ (Fig. 6c).

The intranasal administration of other antigens in combination with flagellin has been shown to significantly increase the antigen-specific IgA titer, not only in the mucosal compartment but also in the serum [10]. Additionally, previous studies found that immunization via the intranasal but not the subcutaneous route elicited sporozoite neutralizing antibodies capable of inhibiting $>90 \%$ of sporozoite invasion [26]. These findings suggest that a comparison of different administration routes of flagellin is needed in future studies.

\section{Conclusions}

We designed a truncated rGP5 gene and prepared HisrGP5, GST-rGP5, and rGP5-FljB proteins with a prokaryotic expression system. The immunoreactivites of His-rGP5 and fusion protein rGP5-FljB were confirmed by western blotting. After the removal of endotoxin in our rGP5 and rGP5-FljB preparations, we verified the activation of an innate immune response via a TLR-5-specific bioactivity assay and investigated the adjuvant activity of FljB via administration of $\mathrm{rGP5}$-FljB protein to mice. Our findings confirm that $\mathrm{FljB}$ could serve as an excellent immunoadjuvant for the production of GP5-specific and PRRSV-specific IgG antibodies and the induction of a robust humoral immune response.

\section{Methods}

\section{cDNA amplification}

Viral RNA was extracted from the Chinese isolate $\mathrm{HH} 08$ of PRRSV using TRIzol reagent (Invitrogen, CA, USA), and viral cDNA was synthesized using Oligo dT primers (Takara, Dalian, China) according to the manufacturer's instructions. Based on the GP5 sequence of PRRSV (GenBank Accession number: GQ184821), primers were designed for amplifying a fragment covering the ORF5 gene of PRRSV (Table 1). PCR was carried out using PrimeSTAR HS DNA Polymerase (Takara) in a $50-\mu \mathrm{l}$ reaction volume consisting of $1 \times$ PrimeSTAR Buffer ( $\mathrm{Mg}^{2+}$ plus), $200 \mu \mathrm{M}$ of each dNTP, $0.2 \mu \mathrm{M}$ of forward and reverse primers, $1.25 \mathrm{U}$ of PrimeSTAR HS DNA Polymerase, and $\sim 200 \mathrm{ng}$ of viral cDNA template. PCR amplifications were performed as follows: 1 cycle of $98^{\circ} \mathrm{C}$ for $5 \mathrm{~min}$, then 25 cycles of $98{ }^{\circ} \mathrm{C}$ for $10 \mathrm{~s}, 60^{\circ} \mathrm{C}$ for $15 \mathrm{~s}$, and $72{ }^{\circ} \mathrm{C}$ for $90 \mathrm{~s}$, followed by 1 cycle of $72{ }^{\circ} \mathrm{C}$ for $10 \mathrm{~min}$. The amplified PCR product was purified, cloned into pCR2.1-T with a TA Cloning Kit (Invitrogen) according to the manufacturer's instructions, and sequenced by GenScript (Nanjing, China). 
Table 1 PCR primers used in this study

\begin{tabular}{lll}
\hline Primer name & Primer sequence $\left(5^{\prime} \rightarrow 3^{\prime}\right)$ & Application \\
\hline GP5-ORF F & ACTTAAGCTTGGTACCATGG & Amplification of GP5 ORF \\
GP5-ORF R & CGCTAGAGCGCTGGCAAGTG & Amplification of GP5 ORF \\
GP5a F & CCGGGATCCAACGCCAGCGACAACAAC & Amplification of GP5a fragment \\
GP5a R & CACCGCCGCTTCCACCGCCACCCTCCACTGCCCAGTCAAA & Amplification of GP5a fragment \\
GP5b F & GTGGAAGCGGCGGTGCGGAAGCTGCATGTCCTGGCGCTA & Amplification of GP5b fragment \\
GP5b R & CCGGAATCGAGACGACCCCATAGTTCCGCT & Amplification of GP5b fragment \\
fljB F & GGGGGATTCGGTGGCGGTGTTCTATGGCACAAGTAATCAACACTAACAGT & Amplification of fljB fragment \\
fljB R & GCGTCGACTTAACGTAACAGAGACAGC & Amplification of fljB fragment \\
\hline
\end{tabular}

\section{Construction of recombinant plasmids}

The GP5 ectodomain with the deletion of its signal peptide and transmembrane regions was designated according to a previously published protocol with minor modifications [5]. The truncated rGP5 was amplified by overlap-PCR with two pairs of primers (Table 1). $B a m \mathrm{HI}$ and EcoRI restriction enzyme sites were introduced in GP5a-F and GP5b-R, respectively (underlined parts). A linker sequence encoding two repeated amino acid sequences (GGGGS) was introduced into primers GP5a-R and GP5b-F (linker sequence in black bold). GP5a and GP5b fragments were amplified by PCR using GP5a-F/GP5a-R and GP5b-F/GP5b-R, respectively, as described above except with an extension for $30 \mathrm{~s}$ at $72{ }^{\circ} \mathrm{C}$. The resulting PCR products were electrophoresed on $1 \%$ agarose gels and purified as the template of the truncated rGP5 gene amplification using primers GP5a-F and GP5b-R. The rGP5 fragment was inserted into the BamHI and EcoRI-digested expression vector pColdI or pGEX-6p-1 to create pCold-rGP5 or pGEX-6p-1-rGP5, respectively. The phase II flagellin $f l j B$ gene was amplified from the genomic DNA of attenuated Salmonella Typhimurium SL7207 strain using primers fljB-F and fljB-R (Table 1). The purified PCR product was digested and cloned into the EcoRI and SalI sites of pCold-rGP5, resulting in a recombinant plasmid pColdrGP5-fljB (Fig. 1). The sequences of the resulting constructs were confirmed by DNA sequencing.

\section{Expression and purification of PRRSV rGP5 and rGP5-FljB}

Recombinant plasmids pCold-rGP5, pGEX-6p-1-rGP5, and pCold-rGP5-fljB were purified with plasmid purification kits (Takara) according to the manufacturer's instructions and transformed into host cells, E. coli, BL21(DE3) pLysS. Protein expression was optimized according to a recently published method with minor modifications [18]. Briefly, the E. coli harboring pCold-rGP5, pGEX-6p-1rGP5, or pCold-rGP5-fljB were cultured in LB liquid medium at $37{ }^{\circ} \mathrm{C}$ with shaking until the optical density (OD) of the culture at $600 \mathrm{~nm}$ reached 0.6. Then, isopropyl- $\beta$-d-thiogalactoside (IPTG) was added to a final concentration of $0.5 \mathrm{mM}$ to induce expression at $25^{\circ} \mathrm{C}$ for $5 \mathrm{~h}$ (pGEX-6p-1-rGP5) or at $15{ }^{\circ} \mathrm{C}$ for $24 \mathrm{~h}$ (pCold-rGP5 and pCold-rGP5-fljB). The empty vector-transformed bacteria were used as a control. The bacteria were pelleted by centrifugation at $10,000 \mathrm{rpm}$, at $4{ }^{\circ} \mathrm{C}$ for $10 \mathrm{~min}$ and re-suspended in TE buffer $(50 \mathrm{mM}$ Tris and $1 \mathrm{mM}$ EDTA, pH 8.0). Next, they were digested with lysozyme at a final concentration of $100 \mu \mathrm{g} / \mathrm{L}$ at room temperature for $30 \mathrm{~min}$. The cell suspension was sonicated on ice for $30 \mathrm{~min}$. Then, the lysate was centrifuged at $10,000 \mathrm{rpm}$ for $10 \mathrm{~min}$ at $4{ }^{\circ} \mathrm{C}$. The supernatant and the pellets were each mixed with sodium dodecyl sulfate (SDS)-loading buffer. Both samples were subjected to $12 \%$ SDS-polyacrylamide gel electrophoresis (SDS-PAGE). The purification of rGP5-FljB was performed by using a His•Bind Purification Kit (Novagen, USA) according to the manufacturer's instructions, and His-rGP5 inclusion bodies were conducted under the condition of $6 \mathrm{M}$ urea. The purified proteins of interest were designated as His-rGP5 or rGP5-FljB. The protein contents were determined with a Bradford assay and by SDS-PAGE analyses.

\section{Western blotting}

The antisera against PRRSV or FljB were generated by immunizing mice with the inactivated PRRSV strain HH08 or FljB protein mixed with Freund's adjuvant. The purified recombinant proteins of rGP5 and rGP5-FljB were both subjected to SDS-PAGE and then transferred to a nitrocellulose membrane. The membranes were blocked with blocking buffer (5\% non-fat dry milk and $0.05 \%$ Tween-20 in phosphate-buffered saline, PBST) at $4{ }^{\circ} \mathrm{C}$ overnight. The next day, the membranes were incubated with a polyclonal antibody against PRRSV or FljB (1:1000 diluted in PBST) at $37^{\circ} \mathrm{C}$ for $2 \mathrm{~h}$. After washing three times with PBST, the membranes were incubated with a horseradish peroxidase (HRP)-conjugated secondary antibody (1:5000 diluted in PBST, Boster, China) at $37{ }^{\circ} \mathrm{C}$ for $1 \mathrm{~h}$. The protein bands were visualized via a diaminobenzidine enzyme-based color development in the dark that was terminated by distilled water. 


\section{Endotoxin removal of rGP5 and rGP5-FljB}

Contaminating lipopolysaccharide (LPS) was removed from the recombinant proteins His-rGP5 and rGP5-FljB by using the ProteoSpin ${ }^{\mathrm{TN}}$ Endotoxin Removal Kit Maxi for protein \& peptides (Norgen, Canada) according to the manufacturer's instructions, and the residual LPS content of the protein was measured using a chromogenic end-point tachypleus amebocyte lysate (CE TAL) assay kit (Chinese Horseshoe Crab Reagent Manufactory Co., Ltd., Xiamen, China) according to the manufacturer's instructions.

\section{TLR-5-specific bioactivity assay}

The detection of IL-8 expression levels in a HEK293mTLR5 cell line (a gift from Dr. Yi Li, Baylor College of Medicine, Houston, USA) was used to test for the TLR5-specific activity of the fusion protein rGP5-FljB. Briefly, HEK293-mTLR5 cells were cultured in 96-well microtiter plates (Corning, USA) at a seeding density of $5 \times 10^{4}$ cells in $100 \mu \mathrm{l} /$ well of DMEM medium supplemented with $10 \%$ fetal calf serum and antibiotics. The next day, cells were treated with endotoxin-free recombinant proteins $\mathrm{rGP} 5$ or $\mathrm{rGP} 5-\mathrm{FljB}$ at concentrations of 10 and $100 \mathrm{ng} / \mathrm{ml}$ for $5 \mathrm{~h}$. For positive controls, HEK293-mTLR5 cells were treated with the TLR-5 agonist flagellin (Sigma-Aldrich, USA). Supernatants were collected and expression levels of IL-8 were then evaluated using Human IL-8 Ready-SET-Go! ELISA Set (eBioscience, San Diego, CA, USA).

\section{Mouse immunization}

Six-week-old female $\mathrm{C} 3 \mathrm{H} / \mathrm{HeJ}$ mice were purchased from the Biomedical Research Institute of Nanjing University. They were housed in isolators and fed a pathogen-free diet and water. The procedures described in this study were approved by the Committee on the Ethics of Animal Experiments of Yangzhou University, Yangzhou, China. To test whether vaccination with the fusion protein rGP5-FljB provides a greater immune response than vaccination with rGP5 alone or with other adjuvants, $\mathrm{C} 3 \mathrm{H} / \mathrm{HeJ}$ mice were randomly divided into five groups (6 mice per group) and immunized intraperitoneally either with rGP5, rGP5-FljB, rGP5 + R848 (Imidazoquinoline; Enzo Life Sciences, USA), rGP5 + aluminum adjuvant (Alum, Thermo, USA), or PBS. These mice were immunized three times on days 0,14 , and 28 at doses of $50 \mu \mathrm{g}$ rGP5, $50 \mu \mathrm{g}$ rGP5-FljB, $10 \mu \mathrm{g}$ R848 or isochoric aluminum adjuvant in $200 \mu \mathrm{L}$. Blood was collected from eye sockets on days 26, 40, 52, and 64 (Fig. 6a) and the sera were stored at $-70{ }^{\circ} \mathrm{C}$ until they were tested by ELISA to determine their antibody levels.

\section{Detection of anti-GP5 and anti-PRRSV antibodies}

Serum IgG, IgG1, and IgG2a titers against GP5 or IgG titers against PRRSV were measured by ELISA according to a previously described protocol with minor modifications [27]. Briefly, 96-well microtiter plates were coated with recombinant GST-GP5 $(0.2 \mu \mathrm{g} / \mathrm{ml})$ or purified inactivated PRRSV strain HH08 $(0.5 \mu \mathrm{g} / \mathrm{ml})$ in $50 \mathrm{mM}$ carbonate buffer $(\mathrm{pH} 9.6)$ at $4{ }^{\circ} \mathrm{C}$ overnight and blocked for $2 \mathrm{~h}$ at $37{ }^{\circ} \mathrm{C}$ with blocking buffer $(1 \%$ bovine serum albumin in PBST). After washing three times with PBST, sera were added at an initial dilution of 1:100 with a two-fold dilution series in blocking buffer and incubated for $2 \mathrm{~h}$ at $37{ }^{\circ} \mathrm{C}$. After five washes with PBST, antigenspecific antibodies were detected using goat anti-mouse IgG conjugated to HRP (1:10,000 dilution) or goat antimouse IgG1 and IgG2a conjugated to HRP (1:3000 dilution) for $1 \mathrm{~h}$ at $37^{\circ} \mathrm{C}$. The ELISAs were developed using $3,3 \neq, 5,5 \neq$-Tetramethylbenzidine and $\mathrm{H}_{2} \mathrm{O}_{2}$ as substrates, and the ODs were read at $450 \mathrm{~nm}$ (A450) with an ELISA reader (Bio-Tek EL 680, USA).

\section{Statistical analysis}

The significance of the difference between groups of fused or mixed proteins and rGP5 alone was determined by a Student's $t$-test with Instat version 5.0 (GraphPad Software, San Diego, CA). Statistical significance was determined at $p<0.05\left(^{(*)}, p<0.01\left(^{(* *)}\right.\right.$ or $p<0.001\left(^{(* * *)}\right.$ ).

\section{Abbreviations}

PRRS: Porcine reproductive and respiratory syndrome; GP5: Glycoprotein 5; PAMPs: Pathogen-associated molecular patterns; TLR-5: Toll-like receptor 5; His: Histidine; IPTG: Isopropyl- $\beta$-d-thiogalactoside; SDS-PAGE: Sodium dodecyl sulfate-polyacrylamide gel electrophoresis; PBS: Phosphate-buffered saline; HRP: Horseradish peroxidase; LPS: Lipopolysaccharide; ELISA: Enzyme-linked immunosorbent assay.

\section{Competing interests}

The authors declare that they have no competing interests.

\section{Authors' contributions}

ZMP and XAJ designed the experiments; DX and LS performed the study; $X Y Z$ analyzed the data; SZG contributed reagents/materials/analysis tools; ZMP, XAJ, DX, and LS wrote the paper. All authors read and approved the final manuscript.

\section{Acknowledgements}

This work was supported by the National Natural Science Foundation of China $(31372415,31172299)$, the Program for New Century Excellent Talents in University (NCET-12-0745), the "Six Talent Peaks Program" of Jiangsu Province (NY-028), the "Qinglan Program" of Jiangsu Province (2012), the Special Fund for Agroscientific Research in the Public Interest (201403054), the National College Students' Innovation and Entrepreneurship Training Programs (20141117008), Jiangsu Province College Students' Practice and Innovation Training Program (201411117008Z) and the Priority Academic Program Development of Jiangsu Higher Education Institutions (PAPD).

Received: 12 February 2015 Accepted: 15 May 2015

Published online: 23 May 2015

\section{References}

1. Wootton SK, Nelson EA, Yoo D. Antigenic structure of the nucleocapsid protein of porcine reproductive and respiratory syndrome virus. Clin Diagn Lab Immunol. 1998;5(6):773-9.

2. Cavanagh D. Nidovirales: a new order comprising Coronaviridae and Arteriviridae. Arch Virol. 1997;142(3):629-33.

3. Dea S, Gagnon CA, Mardassi H, Pirzadeh B, Rogan D. Current knowledge on the structural proteins for porcine reproductive and respiratory syndrome 
(PRRS) virus: comparison of the North American and European isolates. Arch Virol. 2000;145(4):659-88.

4. Plagemann PG. GP5 ectodomain epitope of porcine reproductive and respiratory syndrome virus, strain Lelystad virus. Virus Res. 2004;102(2):225-30.

5. Ren X, Wang M, Yin J, Ren Y, Li G. Heterologous expression of fused genes encoding the glycoprotein 5 from PRRSV: a way for producing functional protein in prokaryotic microorganism. J Biotechnol. 2010;147(2):130-5.

6. Zhao H, Wang Y, Ma Z, Wang Y, Feng WH. Recombinant Kluyveromyces lactis expressing highly pathogenic porcine reproductive and respiratory syndrome virus (HP-PRRSV) GP5 elicits mucosal and cell-mediated immune responses in mice. J Vet Sci. 2013;15(2):199-208.

7. Zuckermann FA, Garcia EA, Luque ID, Christopher-Hennings J, Doster A, Brito $M$, et al. Assessment of the efficacy of commercial porcine reproductive and respiratory syndrome virus (PRRSV) vaccines based on measurement of serologic response, frequency of gamma-IFN-producing cells and virological parameters of protection upon challenge. Vet Microbiol. 2007;123(1-3):69-85.

8. Yin G, Qin M, Liu X, Suo J, Tang X, Tao G, et al. An Eimeria vaccine candidate based on Eimeria tenella immune mapped protein 1 and the TLR-5 agonist Salmonella typhimurium FliC flagellin. Biochem Biophys Res Commun. 2013:440(3):437-42

9. Ricciardi A, Dalton JP, Ndao M. Evaluation of the immune response and protective efficacy of Schistosoma mansoni Cathepsin B in mice using CpG dinucleotides as adjuvant. Vaccine. 2015;33(2):346-53.

10. Lee SE, Kim SY, Jeong BC, Kim YR, Bae SJ, Ahn OS, et al. A Bacterial Flagellin, Vibrio vulnificus FlaB, has a strong mucosal adjuvant activity to induce protective immunity. Infect Immun. 2006;74(1):694-702.

11. Xiong D, Pan Z, Kang X, Wang J, Song L, Jiao X. Molecular cloning and functional analysis of duck Toll-like receptor 5. Res Vet Sci. 2014;97(1):43-5.

12. Mizel SB, Bates JT. Flagellin as an adjuvant: cellular mechanisms and potential. J Immunol. 2010;185(10):5677-82.

13. St Paul M, Brisbin JT, Barjesteh N, Villaneueva Al, Parvizi P, Read LR, et al. Avian influenza virus vaccines containing Toll-like receptors 2 and 5 ligand adjuvants promote protective immune responses in chickens. Viral Immunol. 2014;27(4):160-6.

14. Applequist SE, Rollman E, Wareing MD, Lidén M, Rozell B, Hinkula J, et al. Activation of innate immunity, inflammation, and potentiation of DNA vaccination through mammalian expression of the TLR5 agonist flagellin. J Immunol. 2005;175(6):3882-91.

15. Labarque GG, Nauwynck HJ, Van Reeth K, Pensaert MB. Effect of cellular changes and onset of humoral immunity on the replication of porcine reproductive and respiratory syndrome virus in the lungs of pigs. J Gen Virol. 2000;81(Pt5):1327-34.

16. Ostrowski M, Galeota JA, Jar AM, Platt KB, Osorio FA, Lopez OJ. Identification of neutralizing and nonneutralizing epitopes in the porcine reproductive and respiratory syndrome virus GP5 ectodomain. J Virol. 2002;76(9):4241-50.

17. Yin J, Li G, Ren X, Herrler G. Select what you need: a comparative evaluation of the advantages and limitations of frequently used expression systems for foreign genes. J Biotechnol. 2007;127(3):335-47.

18. Liu B, Li G, Sui X, Yin J, Wang H, Ren X. Expression and functional analysis of porcine aminopeptidase $\mathrm{N}$ produced in prokaryotic expression system. J Biotechnol. 2009;141(1-2):91-6.

19. Li G, Zeng Y, Yin J, Lillehoj HS, Ren X. Cloning, prokaryotic expression, and biological analysis of recombinant chicken IFN-gamma. Hybridoma (Larchmt). 2010;29(1):1-6.

20. Akira S, Hemmi H. Recognition of pathogen-associated molecular patterns by TLR family. Immunol Lett. 2003;85(2):85-95.

21. Boghdadi G, Hammad N, Amer A, Sammour S, Sorour S. R848, a toll-like receptors 7 and 8 agonist, a potential therapy for allergic rhinitis patients. Inflamm Allergy Drug Targets. 2014;13(2):144-9.

22. Lee SE, Koh Yl, Kim MK, Kim YR, Kim SY, Nam JH, et al. Inhibition of airway allergic disease by co-administration of flagellin with allergen. J Clin Immunol. 2008;28(2):157-65.

23. Honko AN, Sriranganathan N, Lees CJ, Mizel SB. Flagellin is an effective adjuvant for immunization against lethal respiratory challenge with Yersinia pestis. Infect Immun. 2006;74(2):1113-20.

24. Gupta SK, Bajwa P, Deb R, Chellappa MM, Dey S. Flagellin a toll-like receptor 5 agonist as an adjuvant in chicken vaccines. Clin Vaccine Immunol. 2014;21(3):261-70

25. Zhang AJ, Li C, To KK, Zhu HS, Lee AC, Li CG, et al. Toll-like receptor 7 agonist imiquimod in combination with influenza vaccine expedites and augments humoral immune responses against influenza A(H1N1)pdm09 virus infection in BALB/c mice. Clin Vaccine Immunol. 2014;21(4):570-9.

26. Carapau D, Mitchell R, Nacer A, Shaw A, Othoro C, Frevert U, et al. Protective humoral immunity elicited by a needle-free malaria vaccine comprised of a chimeric Plasmodium falciparum circumsporozoite protein and a Toll-like receptor 5 agonist, flagellin. Infect Immun. 2013;81(12):4350-62.

27. Cui X, Lei T, Yang D, Hao P, Li B, Liu Q. Toxoplasma gondii immune mapped protein-1 (TgIMP1) is a novel vaccine candidate against toxoplasmosis. Vaccine. 2012;30(13):2282-7.

\section{Submit your next manuscript to BioMed Central and take full advantage of:}

- Convenient online submission

- Thorough peer review

- No space constraints or color figure charges

- Immediate publication on acceptance

- Inclusion in PubMed, CAS, Scopus and Google Scholar

- Research which is freely available for redistribution 